\title{
Posttraumatic stress disorder among adolescents exposed to the ammunition depot blast at Salawa, Sri Lanka - a descriptive study
}

HHS Kaushalya, T Ponnamperuma

\section{Abstract \\ Background}

Beyond the immediate physical consequences of the disaster, trauma exposure may cause lasting mental health problems especially in young people.

Aim

The objective of this study was to investigate the trauma exposure, posttraumatic stress symptoms (PTSS) and the association between trauma exposure and PTSS among the adolescents in Salawa area, who were exposed to the explosion that took place on $5^{\text {th }}$ June 2016 at the ammunition dump of Salawa army camp.

\section{Methodology}

A cross sectional study was conducted eight months after the explosion, among all adolescents in the Salawa ammunition dump area. A self-administered screening instrument was used to assess the nature of the exposure to the blast. The University of California at Los Angeles Posttraumatic Stress Disorder Reaction Index (UCLA PTSD-RI) was used to measure the cumulative trauma exposure and posttraumatic stress symptoms.

Results

A total of 108 adolescents (68.4\% females), with a mean age of $14.3(S D=2.12)$ were included in the survey. Diagnostic criterion for full and partial PTSD was met by $34.2 \%$ and $37.0 \%$ of the participants, respectively. The occurrence of PTSD was significantly associated with the severity of the ammunition blast $(b=3.31, p<0.001)$ and multiple trauma exposure $(b=7.60, p<0.001)$, but was not significantly associated with age or gender.

\section{Conclusions}

There was a relatively high prevalence of PTSD among the adolescents exposed to the blast. Psychological support is needed for these adolescents, and further research is recommended to conduct evidence based interventions in this posttrauma setting.

Keywords: PTSD, trauma, adolescents

SL J Psychiatry 2017; 8(2): 9-13

\section{Background}

An explosion occurred on $5^{\text {th }}$ June 2016, at a key military ammunition storage in Sri Lanka, which stored rockets, mortar, heavy weaponries and other ammunition. This ammunition storage was located in the suburban area of Salawa, in the Awissawella electorate area of the Colombo district. The fire after the explosion lasted overnight, and the explosion affected an area of $8 \mathrm{~km}$ radius from the origin of the blast. According to available data, the explosion claimed the life of one army soldier and caused 47 casualties. Due to this explosion, 1500 houses were damaged and about 7700 families were temporarily shifted to safe places. A peripheral hospital in the area, two factories and many other properties were also destroyed. Beyond the immediate physical consequences of the disaster, trauma exposure may cause lasting mental health problems especially in young people. Posttraumatic stress disorder (PTSD) is a common mental health problem seen among trauma victims. In terms of prevalence, rates of PTSD among adolescents exposed to trauma range from $0 \%$ to $95 \%$ (1). A meta-analysis of PTSD among adolescents reported PTSD rates of $16 \%$ after exposure to any type of trauma, and $10 \%$ following non-interpersonal traumas such as war or natural disasters (1). A longitudinal study in the Gaza strip reported a $41 \%$ prevalence of PTSD among children after exposure to the war (2). In Sri Lanka, Catani et al. (2008) reported a $30 \%$ prevalence of PTSD among children exposed to war and tsunami in the North-East province of the country (3). This observed variation in PTSD prevalence following trauma may be due to variations in the type and severity of the trauma exposure, the time 
since the trauma and other individual and environmental factors may affect the trauma-mental health relationship. These include factors such as the type and magnitude of the disaster, and the age and gender of the exposed individual. In children, exposure to multiple traumatic events is also reported to be a strong risk factor for development of PTSD (4). Among adults, multiple trauma exposure has been associated with experiencing of a longer duration of PTSD symptoms (5).

Evidence on trauma exposure and PTSD among Sri Lankan adolescents is limited. The objective of this study was to describe the prevalence, nature and severity of PTSD among adolescents exposed to the explosion that occurred at the Salawa ammunition storage, and also to explore for factors associated with a higher risk of posttraumatic symptoms. We hypothesized that the presence and severity of PTSD would be associated with the nature and severity of the blast exposure, as well as other factors such as younger age, female gender, and cumulative trauma exposures.

\section{Methodology}

This cross-sectional study was conducted in the Salawa public health midwife (PHM) division, an area that was significantly affected by the Salawa military ammunition depot blast. Data were collected in February 2017, eight months after the event. According to the available statistics at the PHM office Salawa, there were about 1000 people with about 160 adolescents residing in this area at the time of the explosion. We obtained data from all adolescents who remained in the area after the blast, who were aged between 12-18 years. The sample consisted with 108 adolescents. The principal investigator and a trained data collector distributed the selfreported questionnaires to adolescents while at their place of residence - either their homes or at temporary shelters. Participants filled the questionnaire alone, without presence of other family members. They were provided with sufficient time to fill the questionnaires and when needed were provided help by the data collectors.

Ethical approval for this study was granted by the Ethical Review Committee of the Faculty of Medicine, of the University of Ruhuna. Informed consent was obtained from all participants and from their parents, prior to enrolment in the study.

\section{Outcomes of interest and measures used}

The questionnaire consisted of four sections, which examined demographic data, the nature of the ammunition blast exposure, history of previous trauma exposures and PTSD symptoms.

The nature of the blast exposure was measured using a checklist. The checklist consisted of eight questions on blast specific traumatic experience. These questions inquired about the nature of the exposure, as follows: Hearing the explosion close by, hearing the explosion in the distance, directly witnessing the fire of the ammunition depot blast close by, witnessing the blast from a distance, having seen weaponries blasting, having experienced damages to the house due to the blast, having injuries to self by the blast, having injuries to family members or close friends due to the blast, having to leave the house due to the blast. All questions were rated as either $1=y e s$ or $0=$ no. The cumulative score of the above questions ranged from 0 (non- exposure) to 8 (maximum exposure) and this generated the blast exposure severity score for each respondent.

The University of California at Los Angeles Post Traumatic Stress Disorder Reaction Index (UCLAPT SD-RI) was used to screen for PTSD symptoms among adolescents in this study. This questionnaire has not been validated for use among the Sri Lankan adolescent population, but it has been translated to the local language and used by several previous studies, and has good psychometric properties $(6,7)$. The items of this questionnaire are in keeping with the DSM-IV criterion 'A', 'B', 'C' and ' $D$ ', for the diagnosis of PTSD. Criterion ' $A$ ' measured whether the participant appraised the trauma as life threatening event (A1, 7 items) and reacted to it with extreme terror (A2, 6 items). Other items measured re-experiencing of the event (criterion B), avoidance (criterion C) and hyperarousal (criterion D). For a diagnosis of full PTSD all four criterion had to be fulfilled. Respondents who reported a positive criterion A together with any two of the criterion B, C or D were reported to have 'partial PTSD' (8). Adolescents were asked to rate how often they experienced these symptoms during the previous four weeks, on 4-point scales ranging from 0 -never to 4 -most of the time. The cumulative scores of the three clusters B, C and D measured PTSD symptoms severity (PTSS), which ranged from 0-60.

This was measured using the trauma exposure item checklist of the UCLA PTSD-RI. Category A of the PTSDRI explores exposure to traumatic events. This tool measures the experiences of adolescent to 13 different traumas, via $1=y e s$ or $0=$ no responses. The original category "being in a big earthquake that badly damaged the building you were in” was replaced by "experiencing the ammunition blast”. The cumulative trauma exposure was generated by the sum score of 13 different categories of traumas which ranged from 0 - 13 .

\section{Statistical analysis}

Data was analysed using SPSS. Two-tailed $p$-values $\leq 0.05$ considered significant. To address our central hypothesis, we conducted a multiple regression analysis (entry method), considering PTSD symptom severity as the dependent variable. Independent variables age, 
gender (dummy-coded) and age by gender interaction were entered in the first step since these variables have been previously identified by other studies as predictors of trauma exposure and PTSS. We entered blast exposure score and cumulative trauma exposure score in subsequent steps.

\section{Results}

\section{Sample characteristics}

There were 108 participants, of whom $64.8 \%$ were females. The age of the participants ranged between 12-18 years, with a mean of 14.3 years $(\mathrm{SD}=2.12)$. All participants were schooling and most (64.8\%) were in grades 7-10. A majority were Sinhalese and $6.5 \%$ were Tamils. There were 92.6\% Buddhist, 4.6\% Hindus and 2.8\% Christians in the sample.

\section{Exposure to trauma}

All participants had been exposed to the ammunition blast, and the average severity of the ammunition blast exposure was high (mean exposure score $=5.85, S D=1.35$, range 0-8). There was no significant gender difference in exposure to the ammunition blast $\left(M_{\text {girls }}=5.84, S D=1.39\right.$; $\left.\left.M_{\text {boys }}=5.87, S D=1.30\right), t(106)=.09, p=0.93\right)$. Figure 1 gives the experiences reported by participants, during or in the direct aftermath of the blast.

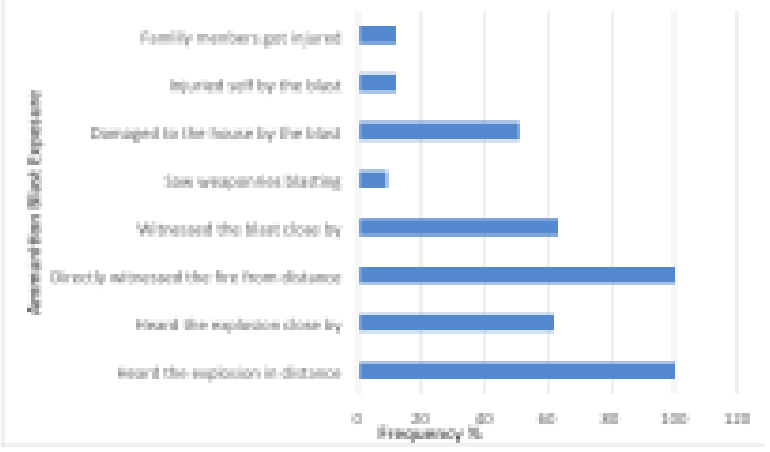

Figure 1. Self-reported participant experiences during or directly after the explosion.

Among the participants, 58.3\% reported exposure to at least one prior traumatic event in their past and the overall multiple trauma exposure (exposure to more than 1 traumatic event) was 58.3\% (Figure 2). The mean trauma exposure was 1.7 ( $S D=1.0$, range $0-12$ ). There was no gender difference in trauma exposure in the sample $\left(\mathrm{M}_{\text {girls }}=1.66, \quad S D=0.97 ; \quad \mathrm{M}_{\text {boys }}=1.73, \quad S D=1.03\right.$, $t(106)=0.35, p=0.73)$. A majority $(n=54)$ of PTSD positive adolescents were females rather than males, but this difference was not statistically significant $\chi^{2}(1, N=108)$ $=1.61 p=.20$ ). A majority (98.1\%) described the Salawa ammunition blast as the worst traumatic experience in their life.

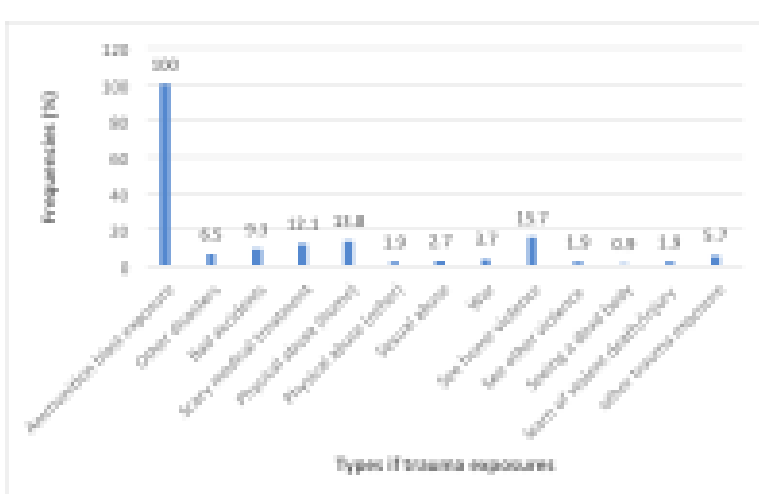

Figure 2. Self-reported exposure to prior trauma.

\section{Posttraumatic Stress Disorder}

As all participants had been exposed to trauma, the UCLA PTSD-RI analysis was conducted for the entire sample. In the study sample $91.6 \%$ participants met the PTSDcriteria A. Additionally, 80.5\%, 48.1\% and $75.0 \%$ of participants met criteria $\mathrm{B}, \mathrm{C}$ and $\mathrm{D}$ respectively. The mean PTSD symptom severity (PTSS) was 20.9 ( $S D=13.8$, range 0-60). PTSS was unrelated to age $r(99)=-.05, p=.61)$. PTSS did not differ by gender $\left(M_{\text {girls }}=14.78\right.$, $S D=12.55 ; \quad M_{\text {boys }}=12.97, \quad S D=12.19, \quad t(97)=0.68$, $p=.50)$. Of the participants, 34.2\% $(n=37)$, met criteria for full PTSD and 37.0\% $(n=40)$ met criteria for partial PTSD.

In order to identify potential predictors of PTSS, we conducted a hierarchical linear regression analysis considering the PTSS score as the dependent variable. Independent variables included the blast exposure score and cumulative trauma exposure score. The model was controlled for age, gender and age by gender interaction (Table 1). The severity of blast exposure, as measured by the blast exposure score, significantly predicted PTSS. Cumulative trauma exposure also significantly associated with PTSS. Age, gender and age by gender interaction showed no significant effect. Model with four variables explained $75.8 \%$ of variance in PTSS.

\section{Discussion}

This study describes the prevalence of PTSD among adolescents, eight months after an ammunition blast in the Salawa area, and explores possible associations between blast exposure and development of PTSD symptoms. 


\begin{tabular}{|c|c|c|c|c|c|c|c|}
\hline Predictor & & B & SEB & $\beta$ & $\mathrm{R}^{2}$ & $\mathrm{R}^{2}$ & Overall F \\
\hline \multicolumn{8}{|l|}{ Model 1} \\
\hline & Age & -0.29 & 0.59 & -0.05 & 0.013 & 0.007 & .349 \\
\hline & Gender & 1.80 & 2.68 & 0.07 & & & \\
\hline & Age by gender & 1.69 & 1.22 & 0.93 & & & \\
\hline \multicolumn{8}{|l|}{ Model 3} \\
\hline & Age & -0.15 & 0.45 & 0.45 & 0.438 & 0.445 & $78.21 * * *$ \\
\hline & Gender & 1.59 & 1.99 & 1.99 & & & \\
\hline & Age by gender & 0.45 & 0.93 & 0.25 & & & \\
\hline & Blast exposure & 6.15 & 0.69 & $0.69 * * *$ & & & \\
\hline \multicolumn{8}{|l|}{ Model 4} \\
\hline & Age & -1.46 & .29 & .03 & .748 & .758 & $117.497^{* * *}$ \\
\hline & Gender & 1.60 & 1.34 & .06 & & & \\
\hline & Age by gender & 0.25 & 0.63 & 0.14 & & & \\
\hline & Blast exposure & 3.31 & .54 & $.36 * * *$ & & & \\
\hline & Cumulative trauma & 7.60 & .70 & $.63 * * *$ & & & \\
\hline
\end{tabular}

Note. The dependent variable is PTSD symptom severity. ${ }^{* * *} p<0.001$

More than half of the participants in this study had experienced the explosion of ammunition very closely and in half of the sample, their houses were damaged. Multiple trauma exposure was reported by more than $50 \%$ of this sample. Ammunition blast exposure and cumulative trauma exposure were more common among males, and reported PTSD symptoms were higher among females, but these differences were not statistically significant.

According to the results of this study, of the 108 adolescent participants, full PTSD was seen in 34.2\%, and partial PTSD in $37.0 \%$. These are relatively high rates of PTSD, in comparison to previous studies from Sri Lanka. For example, a study on war exposed children in Jaffna reported a 25\% PTSD prevalence (9). Catani et al reported 30\% PTSD prevalence in children exposed to war, tsunami and family violence in North East Sri Lanka (3). In another study, adolescents exposed to the tsunami and to a wide variety of other traumas reported a $25 \%$ prevalence of PTSD (7). Studies among adults have also reported similar PTSD prevalence after the trauma exposure (10). The higher levels of full PTSD reported by adolescents in our study may have been due to the severity of the trauma exposure, since the participants had been in the immediate vicinity of the explosion; findings may also have been influenced by the fact that more than half the sample had been exposed to past trauma, which increases the risk of developing PTSD. On the other hand, it is also possible that PTSD symptoms may have been over reported by participants, due to exaggeration of symptoms in anticipation of compensation, since this survey was conducted during the resettlement period after the disaster.

With regards to factors associated with the occurrence of PTSD in this study - age and gender showed no significant association with PTSS in this sample, even though young age and female gender were known risk factors of PTSD (11). This observation is possibly due to the small sample size and narrow age range of adolescents in this study. As expected, more severe trauma exposure and cumulative trauma exposure were associated with more severe PTSS. This finding is in line with previous studies showing trauma exposure to be a significant predictor of PTSD (3). It is also noteworthy that the cumulative trauma exposure was twice as likely to predict PTSS, compared to the blast exposure alone. This suggests that PTSS among adolescents in this study were more strongly associated with cumulative trauma exposure than with blast exposure severity. Therefore, blast experience may have aggravated symptoms related to previous trauma experiences in this sample. However, other factors, such as the time elapsed since exposure to previous traumas, also need to be considered prior to making a definitive conclusion regarding this association.

\section{Limitations}

The present study has number of limitations; first, PTSS were explored by a self-reported questionnaire only; a 
combination of symptom reports by both parents and children in this age group may have yielded more reliable information (12). Second, the high percentage of PTSD cases may reflect some degree of exaggeration of symptoms by adolescents anticipating compensation in the post disaster resettlement period. Further, the UCLA PTSD RI was not a validated questionnaire and this may have influenced our results. Since the study sample was obtained from the blast-affected area, caution is warrant in generalizing the results. Additionally, as some adolescents had left the area after the blast, we were unable to recruit them into the study, and this may have influenced our results. However, every effort was made to mitigate this drawback by obtaining information from all available adolescents currently residing in the area. Lack of information on other factors known to influence trauma - such as social support, and coping styles is a further drawback. Finally, the cross-sectional design of the study precludes causal relationship between the variables.

\section{Conclusions}

There was a relatively high prevalence of PTSD among adolescents exposed to the Salawa ammunition blast, even 8 months after the event. The occurrence of PTSS was significantly associated with the severity of the blast exposure and cumulative trauma exposure, but not with age or gender. These findings suggest that psychological support is needed for these adolescents and further research is recommended to develop evidence-based interventions in this post-trauma setting.

\section{Disclosure statement}

None declared

HHS Kaushalya, Nursing Degree Programme, Faculty of Medicine, University of Ruhuna

T Ponnamperuma, Department of Community Medicine, Faculty of Medicine, University of Ruhuna

Corresponding author: T Ponnamperuma

Email: thejanip@med.ruh.ac.lk

https://orcid.org/0000-0002-0031-348

\section{References}

1. Alisic E, Zalta AK, van Wesel F, et al. Rates of posttraumatic stress disorder in trauma-exposed children and adolescents: meta-analysis. Br J Psychiatry 2014; 204: 335-40.

2. Thabet AA, Vostanis P. Post traumatic stress disorder reactions in children of war: a longitudinal study. Child Abuse Negl 2000; 24(2): 291-8.

3. Catani C, Jacob N, Schauer E, Kohila M, Neuner F. Family violence, war, and natural disasters: a study of the effect of extreme stress on children's mental health in Sri Lanka. BMC psychiatry 2008; 8: 33.

4. Fairbank JA, Fairbank DW. Epidemiology of child traumatic stress. Curr Psychiatry Rep 2009; 11: 289-95.

5. Karam EG, Friedman MJ, Hill ED, et al. Cumulative traumas and risk thresholds: 12-month PTSD in the World Mental Health (WMH) surveys. Depress Anxiety 2014; 31: 130-42.

6. Neuner F, Schauer E, Catani C, Ruf M, Elbert T. Posttsunami stress: a study of posttraumatic stress disorder in children living in three severely affected regions in Sri Lanka. J Trauma Stress 2006; 19(3): 339-47.

7. Ponnamperuma T, Nicolson NA. Negative trauma appraisals and PTSD symptoms in Sri Lankan adolescents. J Abnorm Child Psychol 2016; 44(2): 245-55.

8. Steinberg AM, Brymer MJ, Decker KB, Pynoos RS. The University of California at Los Angeles Post-traumatic Stress Disorder Reaction Index. Curr Psychiatry Rep. 2004; 6: 96-100.

9. Elbert T, Schauer M, Schauer E, Huschka B, Hirth M, Neuner F. Trauma-related impairment in children - a survey in Sri Lankan provinces affected by armed conflict. Child Abuse Negl 2009; 33(4): 238-46.

10. Hollifield M, Hewage C, Gunawardena CN, Kodituwakku P, Bopagoda K, Weerarathnege K. Symptoms and coping in Sri Lanka 20-21 months after the 2004 tsunami. Br J Psychiatry. 2008; 192(1):39-44.

11. Trickey D, Siddaway AP, Meiser-Stedman R, Serpell L, Field AP. A meta-analysis of risk factors for post-traumatic stress disorder in children and adolescents. Clin Psychol Rev. 2012; 32: 122-38.

12. Scheeringa MS, Wright MJ, Hunt JP, Zeanah CH. Factors affecting the diagnosis and prediction of PTSD symptomatology in children and adolescents. Am J Psychiatry. 2006; 163(4): 644-51. 